\title{
Effect of applying saline spray on nasal health status of critically ill patients
}

\author{
Shaimaa Ahmed Awad* \\ Critical Care and Emergency Nursing Department, Faculty of Nursing, Mansoura University, Mansoura, Egypt
}

Received: September 10, 2018

Accepted: December 3, 2018

Online Published: December 18, 2018

DOI: $10.5430 /$ jnep.v9n4p59

URL: https://doi.org/10.5430/jnep.v9n4p59

\begin{abstract}
Objective: A fundamental aspect of hygienic care in ICU is nasal care. However, providing nasal care for critically ill patients is challenging because of caring for very sick patients in a highly stressful environment results in nasal care having a lower priority for nurses than other aspects of care. This study was conducted to investigate the effect of applying saline spray on nasal health status of critically ill patients.

Methods: This study was carried out in intensive care units at Emergency Hospital. Sixty patients were enrolled and divided into a study group who received saline nasal spray three times daily for seven days and a control group who received routine hospital care. Secretion load and nasal cultures were assessed before and on the 7th day of nasal care.

Results: No significance difference was found between control and study groups as regards to secretion score before nasal care. On the 7 th day, there was a statistically significant deterioration of the secretion score by increasing to $3(p<.001)$ in the control group. However, a significant improvement was noted in the study group in comparison to the control group $(p<.001)$. In addition, about half of the control group had proliferated Klebsiella in the first culture which continued to the second culture on the 7th day of routine nasal care while no one had proliferated Klebsiella in the studied sample following administration of normal saline spray which was highly statistically significant.

Conclusions: The use of saline nasal spray is more effective than routine nasal care in reducing colonization with different bacterial species and improving secretion score.
\end{abstract}

Key Words: Nasal spray, Intensive care unit, Saline spray

\section{INTRODUCTION}

Intensive care units (ICUs) have evolved through a growing need to care for critically ill patients with varying dependencies. ${ }^{[1]}$ Hygiene is one aspect of nursing care particularly in ICU as critically ill patients cannot clean their noses and often totally dependent on nursing staff for their personal care. A fundamental aspect of hygienic care in ICU is nasal care. However, providing nasal care for patients in ICU is challenging or even ignored because of caring for very sick patients in a highly stressful environment results in nasal care having a lower priority for nurses than other aspects of medical problems. ${ }^{[2]}$

Moreover, stress factors such as decreased blood pressure, decreased oxygen saturation in the blood or acidosis can cause protein proteolysis that is secreted in the mucosa of the nose predisposing to antimicrobial effect. ${ }^{[3]}$ In addition those critically ill patients are under sedation, which has an effect on the occurrence of hospital-acquired sinusitis. ${ }^{[4]}$

Nasal sprays which composed of saline are often indicated in

\footnotetext{
*Correspondence: Shaimaa Ahmed Awad; Email: shaimaahmed2000@ gmail.com; Address: Critical Care and Emergency Nursing Department, Faculty of Nursing, Mansoura University, Mansoura, Egypt.
}

Published by Sciedu Press 
long term rhino-sinusitis, rhinitis that is caused by sensitive or non-sensitive agents, perforation of septum or following surgery of the nose. Saline nasal sprays help in cleaning of the nose,$^{[5,6]}$ removing of mediators that cause direct inflammation, ${ }^{[7,8]}$ in addition, improving ciliary beat frequency which helps in mucociliary clearance..$^{[9,10]}$ However, less literature is available for detecting the effect of using saline spray on nasal hygiene among critically ill patients. So, the aim of this study was to investigate the effect of applying saline spray on nasal health status of critically ill patients.

\section{MethodS}

A quasi-experimental research design was used to conduct this study. This study was carried out in three intensive care units located at Mansoura Emergency Hospital. ICU1, ICU2 and ICU3. ICU1 and ICU2 each includes 10 beds, 3 mechanical ventilators, 2 crash carts, 2 portable suction machines and 1 defibrillator machine. ICU3 includes 4 beds, 1 mechanical ventilator, 1 crash cart, 1 portable suction and 1 defibrillator machine. Each bed is equipped with advanced technologies such as cardiac monitor, central suction machine and oxygen outlets. A purposive sample of sixty adult critically ill patients were enrolled in the study according to the following inclusion criteria: age range from 21-60 years, expected time in ICU was not less than 7 days, all patients involved in the current study had endotracheal tube, had a nasogastric tube and received antibiotic therapy. Exclusion criteria include patients who were examined and proved to have sinusitis at the time of admission to ICU.

\subsection{Instrumentation}

Two tools were used to collect data in the current study to elicit information about nasal assessment and nasal care performed for critically ill patients. Tool (I): Nasal assessment tool, it composed of 3 parts. Part 1: Patient's characteristics sheet: This included patient's socio-demographic data as well as health relevant data such as underlying disease, medications used, supportive lines and devices and ventilatory modalities. Part 2: Secretion Load sheet: the secretion score which provided by Slapak et al. (2008) ${ }^{[11]}$ which had the following score: (1) refers to absent of secretions; (2) refers to presence of serosal secretions; (3) refers to presence of seropurulent secretions and finally (4) refers to presence of purulent secretions was utilized to evaluate secretion load. Part 3: Nasal culture sheet: culture was obtained from critically ill patients within 24 hours of admission before administering of topical normal saline spray (first culture) and repeated on the 7th day thereafter (second culture). Tool (II): Nasal care tool; nasal care tool was developed by the researcher after reviewing the related literature ${ }^{[12-14]}$ to record nasal activity performed for critically ill patients. It was divided into 2 parts. The first part was assigned for recording nasal suctions before or during nasal care. The second part was assigned for recording nasal care activity which performed 3 times daily for seven days; once every 8 hours using normal saline spray $(0.9 \%)$.

\subsection{Study procedures}

An official written permission to conduct the study was obtained from the responsible authorities at Emergency hospital at Mansoura Main University Hospital, after explanation of the aim of the study. A pilot study was conducted on $10 \%$ of sample to test the applicability of the tools. Appropriate modifications were done prior to data collection for the actual study. Once the necessary approval granted to proceed with the proposed study; data was collected using the designed study tools. Human rights and ethical considerations were kept. On admission, patients were randomly assigned into two groups; control and study groups, each composed of 30 patients. At the beginning, part one of tool (I) was filled in by the researcher to describe patient's general characteristics as well as health relevant data. These include patient's underlying disease, medications used, supportive lines and devices and ventilatory modalities. In addition, a baseline nasal assessment was performed for all patients in the 2 groups using part 2 of tool (I) which described nasal secretion load. Nasal swabs were obtained from all patients recruited for the study in the 2 groups within 24 hours after admission prior to the application of nasal spray and were recorded as baseline data. The cultures were repeated on the 7th day of nasal care. The swabs were transported in a Stuart medium to the laboratory at the Microbiology Diagnostic and Infection Control Unit in the Medical Microbiology and Immunology Department, Faculty of Medicine, Mansoura University. Then, the swabs were removed from the Stuart medium and immersed in 1 milliliter normal saline. Centrifugation for 1 minute was done. Thereafter, quantitative cultures were prepared. For preparing the culture, chocolate agar, 5\% sheep blood agar, and Eosin Methylen-blue Lactose Sucrose agar were used. The cultures were incubated at $37^{\circ} \mathrm{C}$ for $18-24$ hours and assessed the following day. For the study group, 1 puff of nasal spray containing normal saline was administered three times per day (once each shift) for seven days into the nonintubated side. For the control group, routine nasal care was provided by nursing staff which include cleansing of the outer nostrils by tap water. As an evaluation phase, a nasal culture was obtained. To assess the load of secretion, the secretion score provided by Slapak et al. (2008) was used. To investigate the effect of applying saline spray on nasal health status of critically ill patients, comparison was done between the study and control groups regarding secretion score and nasal culture. 


\subsection{Data analysis}

The collected data were coded, organized, categorized, and then transferred into especially designed formats. The statistical analysis of data was done by using SPSS program (statistical package for social science). The data were tabulated and presented. The analysis of the data was performed to test statistical significant difference between variables for both groups (study and control). For quantitative data (mean and standards deviation and Mann-Whitney $U$ test) was used. For qualitative data (frequency and proportion), Chi-square test was used.

\section{RESUlts}

Table 1 illustrates patient's characteristics of the control and study groups: it can be noticed that the mean age of the control and study groups was $44.5 \pm 7.2 \& 44.3 \pm 6.8$ respectively. It can be also noted that the majority of the sample $(83.3 \% \& 80 \%)$ for the control and study groups were on mechanical ventilator. As regards to the underlying disease, head trauma represented $50 \%$ of the control group and $60 \%$ of the study group. No statistically significant difference was elicited between the control and study groups regarding patient's characteristics.

Table 1. Patient's characteristics of the study and control groups

\begin{tabular}{|c|c|c|c|c|c|}
\hline \multirow{2}{*}{ Patients' characteristics } & \multicolumn{2}{|c|}{ Control } & \multicolumn{2}{|c|}{ Study } & \multirow{2}{*}{ Sig } \\
\hline & $\mathbf{N}$ & $\%$ & No & $\%$ & \\
\hline \multicolumn{6}{|l|}{ Gender } \\
\hline Male & 15 & 50 & 17 & 56.7 & $\chi^{2}=0.26$ \\
\hline Female & 15 & 50 & 13 & 43.3 & $p=.60$ \\
\hline Mean age & \multicolumn{2}{|c|}{$44.5 \pm 7.2$} & \multicolumn{2}{|c|}{$44.3 \pm 6.8$} & $\begin{array}{l}t=0.037 \\
p=.94\end{array}$ \\
\hline \multicolumn{6}{|l|}{ Ventilatory modalities } \\
\hline On M.v & 25 & 83.3 & 24 & 80 & $\chi^{2}=0.11$ \\
\hline On T. piece and $\mathrm{O}_{2}$ Source & 5 & 16.7 & 6 & 20 & $p=.73$ \\
\hline \multicolumn{6}{|l|}{ Underlying Disease } \\
\hline $\begin{array}{c}\text { H.T.N } \\
\text { Yes } \\
\text { No }\end{array}$ & $\begin{array}{l}10 \\
20\end{array}$ & $\begin{array}{l}33.3 \\
66.7\end{array}$ & $\begin{array}{l}8 \\
22\end{array}$ & $\begin{array}{l}26.7 \\
73.3\end{array}$ & $\begin{array}{l}\chi^{2}=0.31 \\
p=.57\end{array}$ \\
\hline \multicolumn{6}{|l|}{ Hemiplegic } \\
\hline Yes & 10 & 33.3 & 8 & 26.7 & $\chi^{2}=0.31$ \\
\hline No & 20 & 66.7 & 22 & 73.3 & $p=.57$ \\
\hline \multicolumn{6}{|l|}{ Head trauma } \\
\hline Yes & 15 & 50 & 18 & 60 & $\chi^{2}=0.60$ \\
\hline No & 15 & 50 & 12 & 40 & $p=.43$ \\
\hline \multicolumn{6}{|l|}{ Cerebral Stroke } \\
\hline Yes & 5 & 16.7 & 4 & 13.3 & $\chi^{2}=0.13$ \\
\hline No & 25 & 83.3 & 26 & 86.7 & $p=.71$ \\
\hline \multicolumn{6}{|l|}{ Medications } \\
\hline $\begin{array}{l}\text { Diuretics } \\
\text { Yes } \\
\text { No }\end{array}$ & $\begin{array}{l}25 \\
5\end{array}$ & $\begin{array}{l}83.3 \\
16.7\end{array}$ & $\begin{array}{l}26 \\
4\end{array}$ & $\begin{array}{l}86.7 \\
13.3\end{array}$ & $\begin{array}{l}\chi^{2}=0.13 \\
p=.71\end{array}$ \\
\hline \multicolumn{6}{|l|}{ Anticonvulsants } \\
\hline Yes & 20 & 66.7 & 18 & 60 & $\chi^{2}=0.28$ \\
\hline No & 10 & 33.3 & 12 & 40 & $p=.59$ \\
\hline \multicolumn{6}{|l|}{ Corticosteroids } \\
\hline Yes & 23 & 76.7 & 22 & 73.3 & $\chi^{2}=0.26$ \\
\hline No & 7 & 23.3 & 8 & 26.7 & $p=.76$ \\
\hline
\end{tabular}

Table 2 illustrates comparison between study and control groups as regards to secretion score before and on the 7 th day of nasal care. It can be noted that no significance difference was found between control and study groups as regards to secretion score before nasal care. In addition, it can be seen that, the control group median value was 2 as a baseline data. On the 7 th day, there was a statistically significant deterioration of the secretion score by increasing to $3(p<.001)$. 
A significant improvement was noted in the study group in comparison to the control group $(p<.001)$ on the 7 th day of nasal care.

Table 3 illustrates frequency of nasal colonization with different bacterial species before and seven days after nasal care for the control and study groups: it can be noticed that before nasal care, there was no statistically significant difference between the control and study groups as regards to different bacterial species. In relation to Klebsiella species, in the first culture (before nasal care) $26.7 \%$ of the studied sample had proliferated Klebsiella species which significantly decreased in the second culture that was obtained 7 days after nasal care $(p<.002)$. Moreover, $46.7 \%$ of the control group had proliferated Klebsiella after 7 days of routine care while no one proliferated Klebsiella in the study group after 7 days of nasal care which was highly statistically significance ( $p$ $<.001$ ). On the other hand, 20\% had proliferated Staphylococcus aureus in the control group in the first culture which increased significantly to $46.7 \%$ in the second culture $(p<$ $.02)$. However, in the studied sample only $20 \%$ had proliferated Staphylococcus aureus in the second culture, this difference was statistically significant $(p<.02)$.

Table 2. Comparison between study and control groups as regards to secretion score before and on the 7th day of nasal care

\begin{tabular}{llll}
\hline Groups & Before Nasal Care (baseline) & $7^{\text {th }}$ Day of Nasal Care & Wilcoxon test \\
\hline Control & $2(1-3)$ & $3(2-4)$ & $p<.001$ \\
Median (min-max) & $2(1-3)$ & $2(1-3)$ & $p=.7$ \\
$\begin{array}{l}\text { Study } \\
\text { Median (min-max) }\end{array}$ & $p=.7$ & $p<.001$ & \\
Mann-Whitney U test & & & \\
\hline
\end{tabular}

Note. Secretion score: (1) Absent; (2) Serosal; (3) Seropurulent; (4) Purulent.

Table 3. Frequency of nasal colonization with different bacterial species before and on the 7th day of nasal care for the control and study groups

\begin{tabular}{|c|c|c|c|c|c|c|c|}
\hline \multirow{2}{*}{$\begin{array}{l}\text { Type of Bacterial } \\
\text { Species }\end{array}$} & \multirow{2}{*}{ Time } & & \multicolumn{2}{|c|}{ Control } & \multicolumn{2}{|c|}{ Study } & \\
\hline & & & No & $\%$ & No & $\%$ & \\
\hline \multirow{6}{*}{ Klebsiella } & Before Nasal Care & No & 18 & 60 & 22 & \multirow{2}{*}{73.326 .7} & $\chi^{2}=1.2$ \\
\hline & $\left(1^{\text {st }}\right.$ Culture $)$ & Yes & 12 & 40 & 8 & & $p<.7$ \\
\hline & $7^{\text {th }}$ Day after Nasal Care & No & 16 & 53.3 & 30 & 100 & $\chi^{2}=18.2$ \\
\hline & ( $2^{\text {nd }}$ Culture) & Yes & 14 & 46.7 & 0 & 0 & $p<.001$ \\
\hline & & $\chi^{2}$ & 0.27 & & 9.2 & & \\
\hline & & $p$ & .6 & & .002 & & \\
\hline \multirow{6}{*}{ Staph auras } & Before Nasal Care & No & 24 & 80 & 18 & 60 & $\chi^{2}=2.8$ \\
\hline & $\left(1^{\text {st }}\right.$ Culture $)$ & Yes & 6 & 20 & 12 & 40 & $p<.09$ \\
\hline & $7^{\text {th }}$ Day after Nasal Care & No & 16 & \multirow{2}{*}{53.346 .7} & 24 & 80 & $\chi^{2}=4.8$ \\
\hline & ( $2^{\text {nd }}$ Culture $)$ & Yes & 14 & & 6 & 20 & $p<.02$ \\
\hline & & $\chi^{2}$ & 4.8 & & 2.8 & & \\
\hline & & $p$ & .02 & & .09 & & \\
\hline \multirow{6}{*}{$\begin{array}{l}\text { Alfa hemolytic } \\
\text { streptococci }\end{array}$} & Before Care & No & 26 & \multirow{2}{*}{86.713 .3} & 26 & 86.7 & $\chi^{2}=0$ \\
\hline & $\left(1^{\text {st }}\right.$ Culture $)$ & Yes & 4 & & 4 & 13.3 & $p<1$ \\
\hline & $7^{\text {th }}$ Day after Nasal Care & No & 28 & 93.3 & 30 & 100 & $\chi^{2}=2.07$ \\
\hline & $\left(2^{\text {nd }}\right.$ Culture $)$ & Yes & 2 & 6.7 & 0 & 0 & $p<.15$ \\
\hline & & $\chi^{2}$ & 0.47 & & 4.2 & & \\
\hline & & $p$ & .38 & & .03 & & \\
\hline
\end{tabular}

\section{Discussion}

Nasal care is often ignored in ICU patients as it did not have the main priority of care as other nursing procedures. However, a simple technique can be used for those patients that may be helpful. This technique is the use of nasal spray containing normal saline.
The results of the current study revealed that the mean age of the control and study groups was $44.5 \pm 7.2$ and $44.3 \pm$ 6.3 respectively. Moreover, the majority of the patients were on mechanical ventilation and the most common diagnosis was head trauma. No significant difference was found between the control and study groups as regards to all patients' 
characteristics named gender, age, ventilatory modalities, underlying disease and administered medications.

Also, the results of the current study showed that about one third of the studied sample had proliferated Klebsiella species in the initial culture which significantly decreased in the second culture after administration of saline nasal spray. This result was in line with Senturk et al. (2017) who noted that Klebsiella had proliferated in 7 patients of the studied sample in the first culture but after nasal care only one patient had proliferated Klebsiella. ${ }^{[14]}$

Moreover, in the current study about half of the control group had proliferated Klebsiella in the first culture which continued to the second culture on the 7th day of routine nasal care while no one had proliferated Klebsiella in the studied sample following administration of normal saline spray which was highly statistically significant. This was in line with Ozturan et al. (2017) who stated that the Enterobacteriacea, Klebsiella species that proliferated in the initial culture continued in the last culture in the control group. ${ }^{[14]}$

As regards to Gram positive bacteria named Staphylococcus aureus, about half of the control group had proliferated Staphylococcus aureus in the second culture compared to $20 \%$ in the study group and this difference was significant. This result was in contrast with Senturk et al. (2017) who stated that there was no significant difference between the two groups post care as regards to Gram positive bacteria. In addition no statistical difference was found between the control and study groups as regards to isolation of alfa hemolytic streptococci neither before nor on the 7th day of nasal care. ${ }^{[14]}$

The results of the current study revealed that the use of saline nasal spray is more effective than routine nasal care in reducing colonization with different bacterial species and improving secretion score, these results were consistent with other studies showed that the usage of saline nasal drops 4 times daily through ten days is much helpful than the use of antiobiotic therapy in children with acute sinusitis. ${ }^{[15]}$ It was proven that this therapy decreases such substances as prostaglandins, leukotriene, and histamine which causing direct inflammation. ${ }^{[7]}$ Moreover, it had been applied to decrease allergic and irritated rhinitis symptoms during postoperative period of sinus surgery. ${ }^{[16,17]}$

On the other hand, one of the parameters used for assessing nasal health status in the current study was using secretion load. The secretion score was deteriorated significantly in the control group while a significant improvement was observed in the study group on the 7th day after nasal care. It can be said that this improvement caused by humidification of the nose, dissolving of secretions and activation of mucociliary clearance. This was in line with Ozturan et al. (2017) who stated that in the control group, secretion score increased for 1.7 to 3.1 while in the study group this average decreased from 1.9 to $1.4 .^{[14]}$

\section{SUMMARY}

It can be concluded that the administration of normal saline spray through nostrils for critically ill patients might be recommended as an easy method to improve nasal health status by humidification of the nose, reducing secretion load, and decreasing colonization with different bacterial species.

\section{CONFlicts OF INTEREST Disclosure}

The author declares that there is no conflict of interest.

\section{REFERENCES}

[1] Younes S. Effectiveness of different tracheal tube cuff pressure to minimize aspiration in unconscious patients. Master thesis. Alexandria. University of Alexandria. Faculty of Nursing; 2004.

[2] Fitch J, Munro C, Glass C, et al. Oral Care in adult intensive care unit. Am J Crit Care. 1999; 8(5): 314-318.

[3] Tsang CM, Yip YL, Lo KW, et al. Cyclin D1 expression supports stable EBV infection in nasopharyngeal epithelial cells. Proc. Natl. Acad. Sci. U.S.A. 2012; 109: 3473-3482.

[4] George DL, Falk PS, Umberto Meduri G, et al. Nosocomial sinusitis inpatients in the medical intensive care unit: a prospective epidemiological study. Clin. Infect. Dis. 1998; 27: 463-470.

[5] Karadag A. Nasal saline for acute sinusitis. Pediatrics. 2002; 109: 165. https://doi.org/10.1542/peds.109.1.165

[6] Kurtaran H, Karadag A, Catal F, et al. A reappraisal of nasal saline solutionuse in chronic sinusitis. Chest. 2003; 124: 2036-2037. https://doi.org/10.1378/chest.124.5.2036

Published by Sciedu Press
[7] Georgitis JW. Nasal hyperthermia and simple irrigation for perennial rhinitis: changes in inflammatory mediators. Chest. 1994; 106: 1487-1492. https://doi.org/10.1378/chest.106.5.1487

[8] Ponikau JU, Sherris DA, Kephart GM, et al. Striking deposition of toxic eosinophilmajor basic protein in mucus: implications for chronic rhinosinusitis. J. Allergy Clin. Immunol. 2005; 116: 362-369.

[9] Talbot AR, Herr TM, Parsons DS. Mucociliary clearance and buffered hypertonic saline solution. Laryngoscope. 1997; 107: 500-503. https ://doi.org/10.1097/00005537-199704000-00013

[10] Boek WM, Graamans K, Natzijl H, et al. Nasal mucociliary transport: new evidence for a key role of ciliary beat frequency. Laryngoscope. 2002; 112: 570-573. https://doi.org/10.1097/00005537-2 00203000-00029

[11] Slapak I, Skoupá J, Strnad P, et al. Efficacy of isotonic nasal wash (sea-water) in the treatment and prevention of rhinitis in children. Arch. Otolaryngol. Head Neck Surg. 2008; 134: 67-74. 
[12] Kollef MH, Barie PS. Methicillin-resistant Staphylococcus aureus (MRSA) nasal colonization predicts intensive care unit-acquired MRSA infections: true or false? 2010.

[13] Sarikonda KV, Micek ST, Doherty JA, et al. Methicillin-resistant Staphylococcus aureus nasal colonization is a poor predictor of intensive care unit-acquired methicillin-resistant Staphylococcus aureus infections requiring antibiotic treatment. Critical Care Medicine. 2010; 38(10): 1991-1995. https://doi.org/10.1097/CCM.0b 013e3181eeda3f

[14] Ozturan O, Senturk E, Iraz M, et al. Nasal care in intensive care unit patients. Intensive and Critical Care Nursing. 2017; 44: 36-39. https://doi.org/10.1016/j.iccn.2017.08.006

[15] Topal B, Özsoylu Ş. Are antibiotics required for the treatment of acute sinusitis in children. Yeni Tip Dergisi. 2001; 18(Suppl.): 58-60.

[16] Seppey M, Schweri T, Häusler R. Comparative randomised clini-

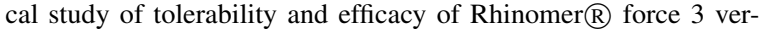
sus a reference product in post-operative care of the nasal fossae after endonasal surgery. ORL. 1996; 58(2): 87-92. https: //doi.org/10.1159/000276805

[17] Holmström M, Rosén G, Wåhlander L. Effect of nasal lavage on nasal symptoms and physiology in wood industry workers. Rhinology. 1997; 35(3): 108-112. 